\section{Microbial characteristics of food preparations in Benevento province}

\author{
Vittoria Ricci, Letizia Petrella, \\ Marika Mercurio, Francesca Barone \\ Agenzia Regionale Protezione Ambiente \\ Campania, Benevento, Italy
}

\section{Abstract}

The aim of this preliminary study was to determine the microbiological quality of pastry products and gastronomic preparations served in food service establishments in Benevento province, Southern Italy. A total of 125 samples were collected from food service establishments. Parameters investigated were: aerobic plate counts (APCs), total Coliform bacteria counts, beta-glucuronidase-positive Escherichia (E.) coli counts, Enterobacteriaceae counts, coagulase-positive Staphylococci counts, isolation of Salmonella spp., Bacillus (B.) cereus counts, and isolation of Listeria (L.) monocytogenes. The microbiological quality was good, with absence of the pathogens $L$. monocytogenes and Salmonella spp. and extremely rare presence of $E$. coli. The fresh pastry and the uncooked gastronomy products were the most contaminated groups; also, cooked cold-served gastronomy products were susceptible to microbiological risk, as a result of the inadequate reheating and the interruption of the warm chain. On the contrary, dried pastry and cooked warm-served gastronomy products showed an excellent hygienic profile. In fact, the amount of compliant samples was $74.4 \%$.

\section{Introduzione}

Negli ultimi trent'anni si è verificata una destrutturazione dell'organizzazione dei pasti consumati nel corso della giornata che sono stati sempre più spesso affiancati da un complesso di altre micro-occasioni di snack (Senauer, 1990) con conseguente variazione della tipologia di alimenti preferiti dai consumatori (Marshall, 1993). Nel periodo 20032009 i pranzi extradomestici sono aumentati del $12,5 \%$, e il mercato della ristorazione - che eroga circa 6,6 miliardi di pasti l'anno - vale oggi 55,4 miliardi di euro (Federazione Italiana Pubblici Esercizi, 2012).

Il presente lavoro ha lo scopo di delineare il profilo igienico-sanitario dei prodotti pronti al consumo somministrati in esercizi di ristorazione della provincia di Benevento, evidenziando al contempo le categorie alimentari maggiormente esposte al rischio microbico e verificando la significatività della differenza di carica microbica riscontrata nei gruppi testati.

Per definire la qualità igienico-sanitaria di tali preparazioni (di pasticceria e gastronomia) sono stati determinati, su campioni di controllo ufficiali prelevati dall'ASL BN1 in fase di preparazione $e$ in fase di vendita al dettaglio, sia indicatori di igiene di processo che di sicurezza alimentare (Reg. CE 2073/2005 integrato dal Reg. CE 1441/2007, DGRC Campania 211/2012 PRI 2011-2014 e Protocollo Tecnico ARPA Piemonte del 2006; Commissione Europea, 2005, 2007; Orsa, 2013).

Inoltre, data la carenza in letteratura di studi relativi al profilo microbico di preparazioni gastronomiche - fatta eccezione per due pubblicazioni dell'Università di Perugia (Cenci-Goga et al., 2005; Favilli et al., 2011) ulteriore obiettivo è stata la produzione, per questa categoria, di valori medi di contaminazione, che potranno servire da riferimento per la messa a punto di protocolli guida per alimenti non sottoposti a specifica normativa di legge (prodotti di gastronomia).

\section{Materiali e Metodi}

Nel semestre settembre 2011-febbraio 2012, l'ASL di Benevento BN 1 ha campionato presso esercizi ristorativi del beneventano 125 alimenti. Questi ultimi sono stati poi trasportati al laboratorio dell'Agenzia Regionale Protezione Ambientale Campania (ARPAC), Dipartimento Tecnico di Benevento, ed analizzati con metodi accreditati dall'ente di certificazione italiano ACCREDIA.

Le matrici alimentari considerate comprendevano sia prodotti di pasticceria che di gastronomia

I prodotti di pasticceria erano ripartiti in due sottogruppi: 25 campioni di pasticceria secca e 25 campioni di pasticceria fresca.

Le preparazioni gastronomiche erano invece suddivise in: 25 preparazioni gastronomiche cotte servite calde; 25 preparazioni gastronomiche cotte servite fredde; e 25 preparazioni gastronomiche crude.

La preparazione dei campioni è stata eseguita conformemente alle indicazioni contenute nelle norme UNI EN ISO 6887-1:2000 (UNI, 2000) e UNI EN ISO 6887-4:2004 (UNI, 2004c).

Per ciascun alimento è stata determinata la carica microbica totale (CMT) a $30^{\circ} \mathrm{C}$ (UNI, 2004a), la conta di Coliformi totali (ISO, 2006), di $E$. coli $b$-glucuronidasi positivo (UNI, 2010a), di Enterobatteriacee (UNI, 2010b), di Stafilococchi coagulasi-positivi (UNI, 2004b), di $B$. cereus presunto (UNI, 2005a), la ricerca di Salmonella spp. (UNI, 2008) e di L. monocytogenes (UNI, 2005b).
Correspondence: Francesca Barone, Agenzia Regionale Protezione Ambiente Campania, via S. Pasquale 38B, 82100 Benevento, Italy. Tel. +39.0824.320217 - Fax: +39.0824.320204

E-mail: f.barone@arpacampania.it

Key words: Food safety, Pastry, Gastronomy, Microbiological analysis.

Received for publication: 15 January 2013.

Revision received: 13 May 2013.

Accepted for publication: 13 May 2013.

This work is licensed under a Creative Commons Attribution 3.0 License (by-nc 3.0).

(C) Copyright V. Ricci et al., 2013

Licensee PAGEPress, Italy

Italian Journal of Food Safety 2013; 2:e28

doi:10.4081/ijfs.2013.e28

Per ogni parametro microbiologico è stata quindi calcolata l'incertezza di misura, così da ottenere un range entro cui si suppone possa cadere il valore vero del misurando: se il limite inferiore dell'intervallo risultava superiore al valore di riferimento si attribuiva al campione un giudizio di non conformità. I valori di riferimento utilizzati sono quelli indicati nel Reg. CE 2073/2005 (Commissione Europea, 2005) così come integrato e modificato dal Reg. CE 1441/2007 (Commissione Europea, 2007), per il solo Bacillus cereus presunto dal DGRC Campania 211/2012 PRI 2011-2014 (Orsa, 2013) e per quanto non riportato da dati di letteratura riassunti nel Protocollo Tecnico ARPA Piemonte del 2006. Per ciascun gruppo campionario, utilizzando il software Statistica (ver.7.0 2004 Stat Soft), sono stati prima determinati parametri di statistica descrittiva, per poi condurre un'analisi di statistica inferenziale (test U di Mann-Whitney), al fine di verificare se le medie dei parametri microbiologici in esame fossero significativamente differenti. A tal fine, per il confronto con la distribuzione normale è stata utilizzata la variabile statistica normale standardizzata $\mathrm{Z}$, adottando 0,05 come P-value.

\section{Risultati}

I risultati dello studio condotto ai fini dell'analisi statistica sono stati espressi in $\log _{10} \mathrm{e}$ riportati in Tabella 1 e Figura 1.

In tutti i prodotti di pasticceria secca erano assenti: $E$. coli b-glucoronidasi positivo, Enterobatteriacee, Salmonella spp., B. cereus e L. monocytogenes. Nella pasticceria fresca 5 campioni erano contaminati da Enterobatteriacee $(0,61 \pm 1,23 \mathrm{Log} \mathrm{UFC} / \mathrm{g})$ e 4 presentavano $B$. cereus $(0,42 \pm 0,98 \mathrm{Log} \mathrm{UFC} / \mathrm{g})$; erano invece sempre assenti $E$. coli b-glucoro- 
nidasi positivo, Salmonella spp. e L. monocytogenes. Tutte le preparazioni gastronomiche cotte servite calde sono risultate negative ad $E$. coli, Enterobatteriacee, Salmonella spp., $B$. cereus e L. monocytogenes. Tra i prodotti gastronomici cotti serviti freddi, invece, un campione era positivo ad Enterobatteriacee $(0,18 \pm 0,91 \mathrm{Log} \mathrm{UFC} / \mathrm{g})$ e 2 a $B$. cereus $(0,19 \pm 0,68 \mathrm{Log} \mathrm{UFC} / \mathrm{g})$. Non è stata riscontrata nessuna contaminazione da $E$. coli b-glucoronidasi positivo, Salmonella spp., e L. monocytogenes. La gastronomia cruda ha presentato 2 campioni positivi ad $E$. coli $(0,13 \pm 0,44 \log$ UFC/g), 8 ad Enterobatteriacee $(1,29 \pm 2,05 \mathrm{Log}$ UFC/g) e 2 a B. cereus $(0,41 \pm 1,43 \mathrm{Log}$ UFC/g); mentre l'intera categoria dei patogeni
Salmonella spp. e L. monocytogenes era assente. Dei 125 campioni esaminati, 32 sono risultati non conformi per almeno un parametro, ovvero il $25,6 \%$ del totale: il $10,4 \%$ di questi era afferente alla pasticceria fresca, il 13,6\% alla gastronomia cruda e l'1,6\% alla gastronomia cotta servita fredda (Figura 2).

Tra i prodotti di pasticceria fresca, 13 erano gli alimenti non conformi. Nell'ambito dei prodotti gastronomici, 17 erano le preparazioni crude non conformi e 2 quelle associate ai prodotti cotti ma somministrati freddi.

Dei 25 campioni di pasticceria fresca, 5 non erano conformi per contenuto in Enterobatteriacee. Tra le preparazioni gastronomiche cotte servite fredde un solo prodotto non era conforme per B. cereus, mentre ben 7 erano le preparazioni gastronomiche crude non conformi per Enterobatteriacee e 2 per B. cereus.

I risultati del test $\mathrm{U}$ hanno confermato, per i parametri CMT e Coliformi e con il rifiuto dell'ipotesi di uguaglianza (Z appartenente all'intervallo $-1,64-+1,64$ ), l'esistenza di una divergenza statisticamente significativa tra la qualità microbiologica della pasticceria secca e fresca (rispettivamente: $\mathrm{Z}=-6,024$ e $\mathrm{P}<0,05$; $\mathrm{Z}=-5,093$ e $\mathrm{P}<0,05)$ tra la gastronomia cruda $\mathrm{e}$ cotta servita fredda (rispettivamente: $Z=-4,579$ e $\mathrm{P}<0,05 ; \mathrm{Z}=-3,114$ e $\mathrm{P}<0,05)$, ma soprattutto tra i prodotti gastronomici crudi e quelli cotti serviti caldi (rispettivamente: $\mathrm{Z}=-6,034$ e $\mathrm{P}<0,05 ; \mathrm{Z}=-4,487$ e $\mathrm{P}<0,05)$.

Tabella 1. Valori medi \pm deviazioni standard per le categorie alimentari indagate.

\begin{tabular}{|c|c|c|c|c|c|}
\hline & $\begin{array}{l}\text { Pasticceria } \\
\text { secca }\end{array}$ & $\begin{array}{l}\text { Pasticceria } \\
\text { fresca }\end{array}$ & $\begin{array}{l}\text { Gastronomia cotta } \\
\text { servita calda }\end{array}$ & $\begin{array}{c}\text { Gastronomia cotta } \\
\text { servita fredda }\end{array}$ & $\begin{array}{l}\text { Gastronomia } \\
\text { cruda }\end{array}$ \\
\hline $\begin{array}{l}\text { CMT } \\
\text { (Log UFC/g) }\end{array}$ & $1,80 \pm 1,03$ & $4,85 \pm 1,32$ & $0,98 \pm 1,20$ & $2,80 \pm 1,78$ & $5,14 \pm 1,12$ \\
\hline $\begin{array}{l}\text { Coliformi } \\
\text { (Log UFC/g) }\end{array}$ & - & $2,72 \pm 1,71$ & - & $0,37 \pm 0,79$ & $2,68 \pm 2,43$ \\
\hline $\begin{array}{l}\text { E. coli } \\
\text { (Log UFC/g) }\end{array}$ & - & - & - & - & $0,13 \pm 0,44$ \\
\hline $\begin{array}{l}\text { Enterobatteriacee } \\
\text { (Log UFC/g) }\end{array}$ & - & $0,61 \pm 1,23$ & - & $0,18 \pm 0,90$ & $1,29 \pm 2,05$ \\
\hline $\begin{array}{l}\text { Stafilococchi } \\
\text { (Log UFC/g) }\end{array}$ & - & $0,51 \pm 1,20$ & - & $0,05 \pm 0,26$ & $0,24 \pm 0,67$ \\
\hline $\begin{array}{l}\text { Salmonella spp. } \\
\text { (Log UFC/g) }\end{array}$ & - & - & - & - & - \\
\hline $\begin{array}{l}\text { B. cereus presunto } \\
\text { (Log UFC/g) }\end{array}$ & - & $0,42 \pm 0,98$ & - & $0,19 \pm 0,68$ & $0,41 \pm 1,43$ \\
\hline $\begin{array}{l}\text { Listeria m. } \\
\text { (Log UFC/g) }\end{array}$ & - & - & - & - & - \\
\hline
\end{tabular}

CMT, carica microbica totale; E. coli, Escherichia coli; Salmonella spp., Salmonella species; B. cereus presunto, Bacillus cereus presunto; Listeria m., Listeria monocytogenes.

\section{DISTRIBUZIONE DEI CAMPIONI NON} CONFORMI NEI DIVERSI GRUPPI

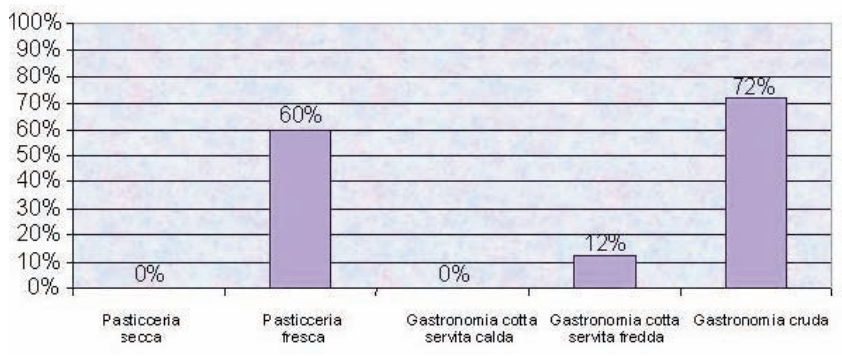

Figura 1. Rappresentazione grafica, per categoria alimentare d'appartenenza, della percentuale di campioni con uno o più parametri microbiologici superiori al rispettivo valore limite/guida, sottoposti a determinazione dell'incertezza di misura.
PERCENTUALE DEI CAMPIONI CONFORMI E NON PER CATEGORIA ALIMENTARE

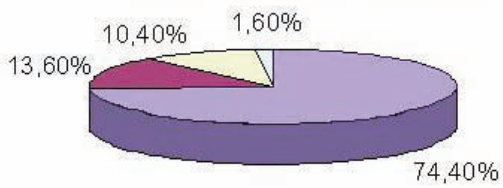

$\square$ CONFORMI

- NON CONFORMI GASTRONOMIA CRUDA

$\square$ NON CONFORMI PASTICCERIA FRESCA

口NON CONFORMI GASTRONOMIA COTTA SERVITA FREDDA

Figura 2. Rappresentazione grafica delle conformità e delle non conformità complessive, quest'ultime ripartite per categoria alimentare d'appartenenza. 
della Commissione del 5 dicembre 2007 che modifica il regolamento (CE) $n$. 2073/2005 sui criteri microbiologici applicabili ai prodotti alimentari, 1441/2007/CE. In: Gazzetta Ufficiale, L 322/12, 07/12/2007.

Dallo studio condotto si evince che la categoria alimentare maggiormente esposta al rischio microbiologico è rappresentata dalla gastronomia cruda. Difatti, il mancato trattamento termico non consente l'abbattimento della carica batterica che può interessare l'alimento nelle diverse fasi della sua filiera produttiva.

Il pericolo può riguardare, sebbene in misura inferiore, anche i cibi cotti serviti freddi, a causa dell'interruzione della catena del caldo e/o di una cottura inadeguata, che non consente il raggiungimento di $75^{\circ} \mathrm{C}$ al cuore dell'alimento per un tempo sufficientemente esteso.

Altra categoria alimentare esposta a contaminazione batterica è la pasticceria fresca, a causa di materie prime (panna e uova) utilizzate per la preparazione di farciture che possono essere contaminate già all'origine. Inoltre, le caratteristiche di composizione, umidità e $\mathrm{pH}$ delle creme, la pratica di produzione spesso manuale e l'inosservanza di un'accurata igiene possono costituire ulteriori cause di proliferazione microbica.

Il numero di campioni esaminato non è tale da permettere di considerare i risultati ottenuti fruibili per l'emanazione di nuovi protocolli guida per alimenti per i quali non esiste una specifica norma di riferimento, ma vuole essere uno studio preliminare che serva ad incentivare la produzione di nuovi dati in questo settore alimentare.

\section{Bibliografia}

Cenci-Goga BT, Ortenzi R, Bartocci E, Codega de Oliveira A, Clementi F, Vizzani A, 2005. Effect of the implementation of HACCP on the microbiological quality of meals at a university restaurant. Foodborne Pathog Dis 2:138-45.

Commissione Europea, 2005. Regolamento della Commissione del 15 novembre 2005 sui criteri microbiologici applicabili ai prodotti alimentari, 2073/2005/CE. In: Gazzetta Ufficiale, L 338/1, 22/12/2005.

Commissione Europea, 2007. Regolamento
UNI, 2004b. Microbiologia di alimenti e mangimi per animali. Metodo orizzontale per la conta di stafilococchi coagulasi-positivi. Tecnica che utilizza il terreno agar BairdParker. Norma UNI EN ISO 6888-1:2004. Ente Nazionale Italiano di Unificazione ed., Milano, Italia.

UNI, 2004c. Norma UNI EN ISO 6887-4:2004. Preparazione dei campioni di prova, sospensione iniziale e diluizioni decimali per l'analisi microbiologica. Parte 4: regole specifiche per la preparazione di prodotti diversi dal latte e dai prodotti del latte, dalla carne e prodotti a base di carne, dal pesce e prodotti della pesca. Ente Nazionale Italiano di Unificazione ed., Milano, Italia.

UNI, 2005a. Microbiologia di alimenti e mangimi per animali. Metodo orizzontale per la conta di Bacillus cereus presunto. Tecnica della conta delle colonie a $30^{\circ} \mathrm{C}$. Norma UNI EN ISO 7932:2005. Ente Nazionale Italiano di Unificazione ed., Milano, Italia.

UNI, 2005b. Microbiologia di alimenti e mangimi per animali. Metodo orizzontale per la ricerca e la conta di Listeria monocytogenes. Norma UNI EN ISO 11290-2:2005. Ente Nazionale Italiano di Unificazione ed., Milano, Italia.

UNI, 2008. Microbiologia di alimenti e mangimi per animali. Metodo orizzontale per la ricerca di Salmonella spp. Norma UNI EN ISO 6579/2008. Ente Nazionale Italiano di Unificazione ed., Milano, Italia.

UNI, 2010a. Microbiologia di alimenti e mangimi per animali. Metodo orizzontale per la conta di Escherichia coli beta glucoronidasi-positiva. Parte 2: tecnica della conta delle colonie a $44^{\circ} \mathrm{C}$ che utilizza 5 -bromo4-cloro-3-indolil beta-D-glucoronide. Norma UNI EN ISO 16649-2:2010. Ente Nazionale Italiano di Unificazione ed., Milano, Italia.

UNI, 2010b. Microbiologia di alimenti e mangimi per animali. Metodo orizzontale per la ricerca e la conta di Enterobatteriacee. Parte 2: Metodo della conta delle colonie. Norma UNI EN ISO 21528-2:2010. Ente Nazionale Italiano di Unificazione ed., Milano, Italia. 